Research Article

\title{
The BER of the New FSO Receiver Manufactured by RTCVD and Solar Cell Technology
}

\author{
Qinggui Hu iD $^{1,2}$ and Long Wenguang ${ }^{1}$ \\ ${ }^{1}$ Information Construction and Service Center, Neijiang Normal University, Neijiang, Sichuan 641100, China \\ ${ }^{2}$ Solar Energy Integration Technology Popularization and Application Key Laboratory of Sichuan Province, Panzhihua, \\ Sichuan 641100, China \\ Correspondence should be addressed to Qinggui Hu; hu646100178@126.com
}

Received 26 May 2020; Revised 28 September 2020; Accepted 29 October 2020; Published 7 November 2020

Academic Editor: E. Bernabeu

Copyright (c) 2020 Qinggui Hu and Long Wenguang. This is an open access article distributed under the Creative Commons Attribution License, which permits unrestricted use, distribution, and reproduction in any medium, provided the original work is properly cited.

\begin{abstract}
In the free-space optical (FSO) communication system, alignment and coupling are key issues. In this work, we adopt a PIN photodiode board as the new receiver to address this question. Firstly, with rapid thermochemical vapor deposition (RTCVD) and solar cell technology, the PIN photodiode board is manufactured. Then, using scanning electron microscope (SEM) and transmission electron microscope (TEM), the microphotographs of the PIN photodiode are taken. After that, the PIN board is arranged as a new receiver in the FSO system to do a bit error rate (BER) experiment. In total, we have carried out 4 groups of experiments. The BERs of the ordinary receiver are as follows: $\left(10^{-8.5}, 10^{-8}, 10^{-8}\right.$, and $\left.10^{-7.9}\right)$ and that of the new receiver is $\left(10^{-9.2}\right.$, $10^{-9.1}, 10^{-9.1}$, and $\left.10^{-9}\right)$, respectively. It means the BER of the new receiver is lower. In other words, the new receiver performs better.
\end{abstract}

\section{Introduction}

FSO communication has attracted growing interests of scholars and researchers because of its advantages such as fast transmission rate and wide space coverage. In FSO, the optical antennas, as a part of receiving system, usually including a series of lenses, focus optical signals into an optical fiber. Optical fiber transmits the optical signals to PIN/APD diodes, where the optical signal is converted to an electrical signal.

In such systems, the coupling of light-into-fiber is the key issue. Many documents have studied this question. In [1], it focuses on neighbor discovery using high-directional transceivers over the same communication channel. In [2], an adaptive digital combination algorithm for coherent FSO communication based on binary phase-shift keying (BPSK) and orthogonal phase-shift keying (QPSK) modulation is proposed to eliminate the time-consuming and complex estimation process of random time-varying channel fading. In [3], the performance of FSO communication system using a PIN photodiode receiver for $M$-element phase-shift keying (PSK) is evaluated under the situation of strong non-Kolmogorov atmospheric turbulence. In [4], the average capacity of free-space optical communication over a Malaga atmospheric turbulent channel with pointing error and path loss is studied for intensity modulation/direct detection (IM/ DD) and heterodyne detection. In [5], the performance of multiband phase shift keying (PSK) SIM communication system using PIN photodiode receivers is evaluated under strong non-Kolmogorov atmospheric turbulence with Gauss beam as an excitation source.

In our previous work, we have studied this question and have published several papers. In [6], a new type of conical optical receiver is proposed, in which a special tapered structure can improve the coupling efficiency by enlarging the optical receiving area. In [7], the conical array is proposed as a new receiver to improve efficiency and the sample of the conical arrays was fabricated in the laboratory. In [8], the conical fiber arrays were fabricated in the laboratory and test experiment under microvibration environment is done 
to compare its performances with an avalanche photodiode (APD). In [9], in order to analyze the loss of free space optical receiver caused by vibration, coordinate systems are established on the surface of receiving lens and receiving optical fiber, respectively. Then, using Gauss optical theory, the coupling efficiency equation is obtained. In [10], the error rate and coupling characteristics of the new tapered fiber have been studied. In [11], a PIN photodiode array is proposed to receive space light directly.

On the whole, in above approaches, for the receiving system, the problem of too small acceptance area has not been solved well. Then, the alignment and track become a great challenge. Especially, the problem will become worse when the distance is long.

In this work, we attempt to address this question from a new way. We adopt a particular PIN board as the receiver, which is manufactured with solar cell technology. Since the PIN board can convert the optical signal into electrical signal directly, then the coupling of light-into-fiber is not needed. Because the receiving area of PIN board is large, the difficulty in alignment is reduced greatly. In above approaches, some of them have studied the PIN photodiode receiver. However, our work is different. The feature of our work is adopting solar cell technology to produce the PIN board. With the vigorous development of solar energy industry, the solar cell technology becomes more and more advanced. In this paper, we work with the technicians of Zhejiang Anxun Solar Energy Technology Co., Ltd. to produce the PIN board. After the sample of the PIN board is produced, its I-V curves are tested. Then, it is arranged in FSO system to do the experiment. The experimental result shows its BER is lower than that of the traditional receiver.

\section{Typical and New Spatial Optical Communication System}

Figure 1(a) shows a typical FSO system. Laser beams are coupled into fiber through the antenna and converted into electrical signal in the PIN detector [12-14]. Figure 1(b) shows the new FSO system, in which the PIN board receives optical signal and converts the optical signal into electrical signal directly. In the new system, the optical antenna system is not needed.

\section{Manufacture of PIN Board}

3.1. The Making Process of the Sample. In recent years, with the high-speed development of the solar energy industry, the producing process of solar cells becomes more and more sophisticated $[15,16]$. In order to produce the PIN board, we conduct the research with the technicians of Zhejiang Anxun Solar Energy Technology Co., Ltd. This company is located in Yiwu City, Zhejiang Province, China. It is mainly engaged in research, manufacturing, sales, and after-sales service of crystalline silicon solar cells. After several months of effort, the $p$-Ge/i-Ge layer is grown on the phosphorus-doped $n$ type $\mathrm{Si}$ wafer with the solar cell technology and RTCVD technology. It means the sample is fabricated successfully. RTCVD is a mature technology (for more details, refer
[17-20]). The manufacture process includes the following steps:

(a) Detection and clean. The phosphorus-doped n-type Si wafer is the carrier of PIN cells. It is necessary to detect the parameters such as minority electron lifetime and resistivity to eliminate the unqualified silicon wafers. Then, the wafers are wet cleaned with HF (HF: DI = 6:1).

(b) Growth of Ge buffer layers on the phosphorusdoped $n$-type $\mathrm{Si}$ wafer. We use the equipment ECOPIA-RTCVD-100 to do this work; it is the product of ECOPIA Company, South Korea. More information can be found on its website and the product manual. In this process, the main parameters are as follows: temperature: $350^{\circ} \mathrm{C}$; pressure: 20 Torr; source gas: $\mathrm{GeH}_{4}\left(20 \%\right.$ in $\left.\mathrm{H}_{2}\right)$; and thickness of Ge buffer layers: $\sim 110 \mathrm{~nm}$.

(c) Growth of high temperature i-Ge layers. In this process, the main parameters are as follows: temperature: $500^{\circ} \mathrm{C}$; pressure: 20 Torr; source gas: $\mathrm{GeH}_{4}$ $\left(20 \%\right.$ in $\left.\mathrm{H}_{2}\right)$ at $30 \mathrm{sccm}$ with $20 \mathrm{slm} \mathrm{H}_{2}$ as carrier gas; and thickness of $\mathrm{i}-\mathrm{Ge}$ layers: $\sim 1.4 \mu \mathrm{m}$.

(d) Growth of boron-doped p-type Ge layers. The doping concentration is about $10^{18} \mathrm{~cm}^{-3}$. In this process, the main parameters are as follows: temperature: $500^{\circ} \mathrm{C}$; pressure: 20 Torr; source gas: $\mathrm{GeH}_{4}$ $\left(20 \%\right.$ in $\left.\mathrm{H}_{2}\right)$ at $30 \mathrm{sccm}$ with $20 \mathrm{slm} \mathrm{H}_{2}$ as carrier gas; and thickness: $\sim 0.32 \mu \mathrm{m}$.

(e) Growth of synthesis of boron-doped Ge layer. In this process, $100 \mathrm{ppm}_{2} \mathrm{H}_{6}$ in $\mathrm{H}_{2}$ is used with 0.025 of the dopant number. The main parameters are as follows: temperature: $500^{\circ} \mathrm{C}$; pressure: 20 Torr; source gas: $\mathrm{GeH}_{4}\left(20 \%\right.$ in $\left.\mathrm{H}_{2}\right)$ at $30 \mathrm{sccm}$ with $20 \mathrm{slm}$ $\mathrm{H}_{2}$ as carrier gas.

(f) In this stage, the $p$-Ge/i-Ge layer is grown on $\mathrm{Si}$ wafer. Then, in order to check the hierarchical structure, we use both SEM and AFM to measure the thickness of the $p$-Ge/i-Ge layer. To ensure the diameters of the mesas ranging from $100 \mathrm{~mm}$ to $120 \mathrm{~mm}$, we use a load-locked BMR (HiEtch) highdensity plasma etch system to etch the sample $[21,22]$. The etch system consists of chamber (operating at $2 \mathrm{MHz}$ ), an inductively coupled plasma (ICP), and an additional RF bias (13.56 MHz).

(g) Deposition of $\mathrm{Si}_{3} \mathrm{~N}_{4}$ of $4000 \AA$ on the $n$-Ge/i-Ge layer. For this purpose, plasma-enhanced chemical vapor deposition (PECVD) is used. After that, contact metallurgy of $\mathrm{Ni}(300 \mathrm{~nm}) / \mathrm{Au}(300 \mathrm{~nm})$ is deposited by electron-beam evaporation. After fabrication, the contacts are treated by rapid thermal annealing at $500^{\circ} \mathrm{C}$ for $30 \mathrm{~s}$ in an $\mathrm{N}_{2}$ environment.

(h) Silk-screen printing. In this stage, PIN junctions can generate current under light. It is necessary to fabricate positive and negative electrodes on the surface of PIN cells. There are many ways to make electrodes. Among them, silk-screen printing is the 


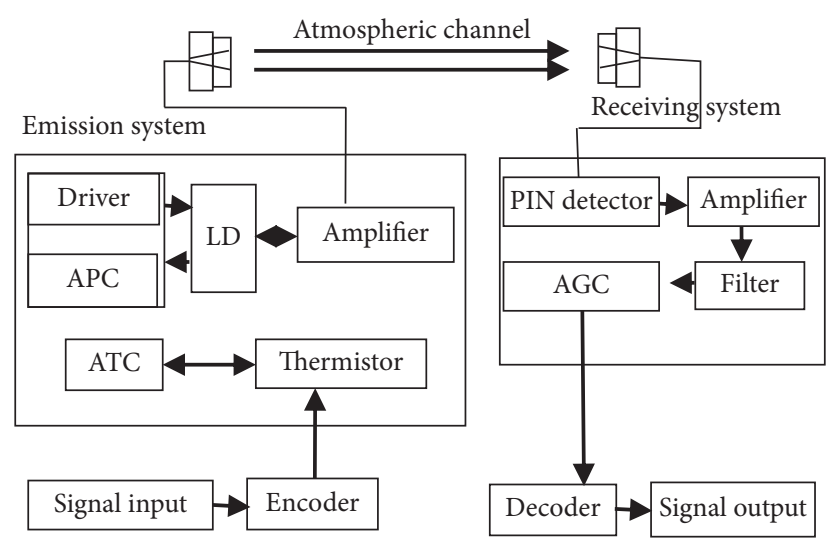

(a)

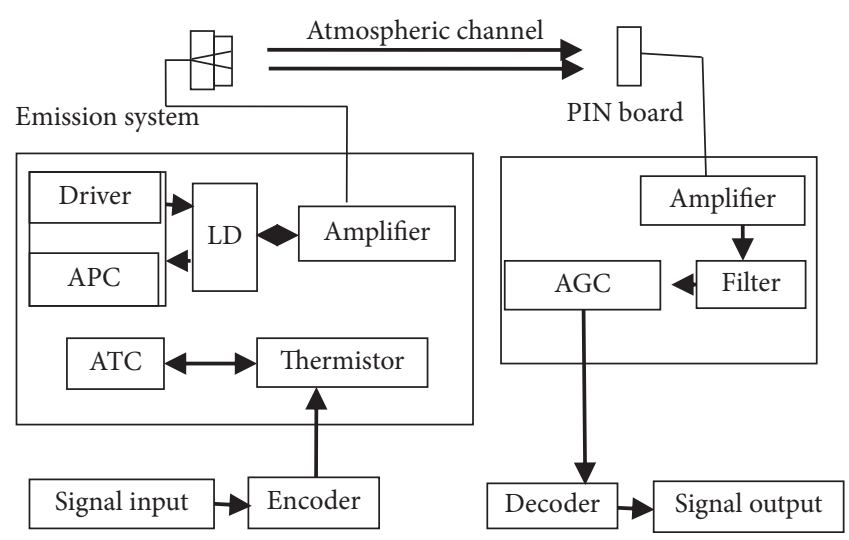

(b)

Figure 1: The space optical communication system: (a) the typical system; (b) the new system.

most common way. Screen printing is to print predetermined graphics on the substrate by means of imprinting. The equipment consists of three parts: silver-aluminum slurry printing on the back of the battery, aluminum slurry printing on the back of the battery, and silver slurry printing on the front of the battery.

(i) Rapid sintering. In this stage, the PIN cells need to be sintered quickly in a sintering furnace to burn off the organic resin binder, leaving almost pure silver electrodes which are tightly bonded to the wafer. The sintering furnace is divided into three stages: presintering, sintering, and cooling. The purpose of the presintering stage is to decompose and burn the high-polymer binder, and the temperature rises slowly in this stage. During the sintering stage, various physical and chemical reactions are completed to form a resistance film structure so that it has resistance characteristics, and the temperature reaches a peak value in this stage. During the cooling stage, the resistance film structure is fixed on the substrate.

In above steps, (b), (c), (d), (e), and (f) belong to RTCVD technology, while (g), (h), and (i) belong to common technology of solar cells. In fact, the RTCVD technology is similar to both low-pressure chemical vapor deposition (LPCVD) and plasma-enhanced chemical vapor deposition (PECVD). Both LPCVD and PECVD are common technologies in solar cell industry.

3.2. The Discussion about the Sample. After the new sample of PIN board is produced, we have measured it. Its shape is about $10 \times 8 \mathrm{~cm}^{2}$. Figure 2 shows the surface morphology by using scanning electron microscope (SEM). Figure 3 shows the cross-sectional image by using transmission electron microscope (TEM). The inset of Figure 3 is the enlarged image of the interface.

For the ideal diode, the forward-biased current can be expressed as follows [23, 24]:

$$
\begin{gathered}
I=I_{\mathrm{s}}\left[\exp \left(\frac{\mathrm{qV}}{\mathrm{nkT}}\right)-1\right], \\
n=\frac{q \mathrm{~d} V}{k T \mathrm{~d}(\ln I)} .
\end{gathered}
$$

In above equations, $n$ is known as the ideal factor, and it is a quantity parameter; $k$ is the Boltzmann constant; $T$ is the absolute temperature; $V$ is the positive bias voltage; and $I_{s}$ is the reverse saturation.

In view of the Shockley theory, at a low voltage, $n$ is about 1.0. At a higher voltage, it is up to 2.0. However, this theory is not applicable to the situation when $n$ is greater than 2.0. For such case, equation (1) needs to be modified as follows $[25,26]$ :

$$
I-\frac{V-\mathrm{IR}_{s}}{R_{\mathrm{p}}}=I_{\mathrm{s}}\left[\exp \left(\frac{q\left(V-\mathrm{IR}_{s}\right)}{n k T}\right)-1\right],
$$

where $R_{p}$ is the parallel resistance and $R_{s}$ is the series resistance. When $R_{p}$ is towards infinity, $R_{s}$ is towards 0 .

In such case, equation (3) reduces to equation (1).

The parallel resistance $\left(R_{p}\right)$ can be deduced near the I-V curve origin, where $\left(V<<\left(E_{\mathrm{g}} / q\right)\right)$ :

$$
R_{p}=\left.\frac{\mathrm{d} V}{\mathrm{~d} I}\right|_{\text {near origin }} .
$$

In general, the series resistance is much smaller than parallel resistance, namely, $R_{s}<<R_{p}$. Then, we can evaluate the series resistance at a high voltage without taking the parallel resistance into account. The high voltage means the voltage exceeds turn-on limit, namely, $V>\left(E_{\mathrm{g}} / q\right)$. In such case, the I-V curve becomes linear, and $R_{s}$ is given by

$$
R_{s}=\left.\frac{\mathrm{d} V}{\mathrm{~d} I}\right|_{\text {voltages exceeding turn-on }} \text {. }
$$

For our pin photodiode, we have done the experiment to test its I-V characteristics at dark. The result is shown in Figure 4 , in which both $R_{p}$ and $R_{s}$ are marked. From this figure, we can see the current is allowed to flow only in positive direction and the reverse current is close to zero. 


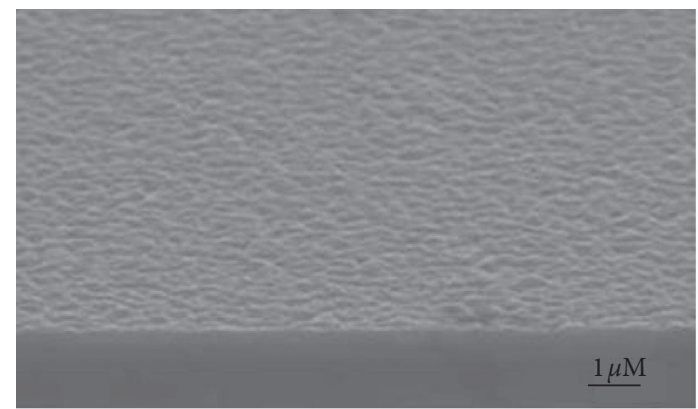

Figure 2: Surface morphology by SEM.

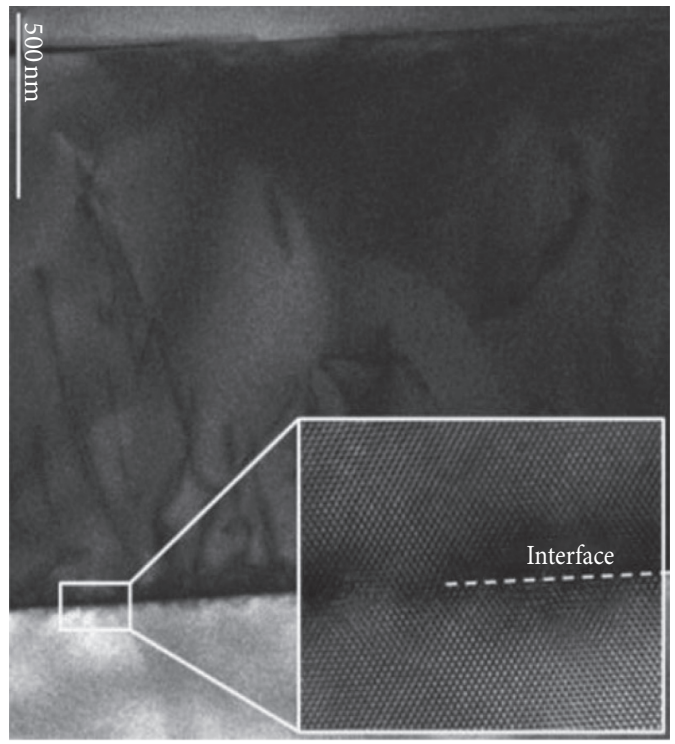

FIgURE 3: Cross-sectional image by TEM.

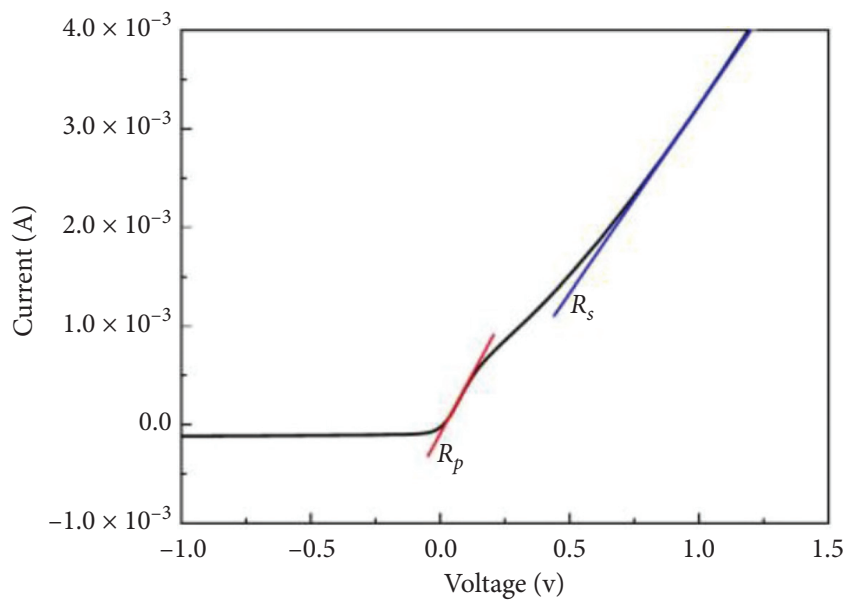

FIgURE 4: I-V curves of pin photodiode at dark.

At the same time, we have measured the photocurrent $\left(I_{\mathrm{ph}}\right)$ under the illumination of different wavelengths: $1.10 \mu \mathrm{m}, 1.31 \mu \mathrm{m}$, and $1.55 \mu \mathrm{m}$. As shown in Figure 5, the responsive photocurrents of $1.10,1.31$, and $1.55 \mu \mathrm{m}$ are 0.38 , 0.23 , and $0.19 \mathrm{~A} / \mathrm{W}$, respectively. Indeed, $I_{\mathrm{ph}}$ could be roughly estimated by the Hecht formula [27-29]:

$$
\frac{I_{\mathrm{ph}}}{I_{\mathrm{sat}}}=\frac{\mu \tau V_{b}}{\mathrm{~d}^{2}}\left[1-\exp \left(\frac{-\mathrm{d}^{2}}{\mu \tau V_{b}}\right)\right],
$$

where $\mu \tau, \mathrm{d}$, and $I_{\text {sat }}$ are the effective life, $i$-layer thickness, and the saturation photocurrent, respectively. $V_{b}=V+V_{i}$, where $V$ is the applied voltage and $V_{i}$ is the built-in voltage of the photodiode. If there is even electric field in the device, and only one trapping level is in consideration, we can use equation (6) to estimate Iph for the first approximation.

\section{The Experiment}

In order to study the BER performance of the PIN board in the FSO system, we do the following experiment. As shown in Figure 6(a), it adopts an ordinary receiving system in FSO [22]. In the experiment, the bit error tester produces signals and radiates them. After the signals go through the vibration deflector, they are coupled into fiber by the receiving lens. Then, the optical signals are converted into electrical signals in PIN [23]. In the next step, after the electrical signals are processed by the signal processor, they come back to the bit error tester. In the experiment, the vibration environment is used to simulate the alignment error.

While, in Figure 6(b), we adopt a new receiving system in FSO, where the PIN board receives the optical signals and converts them into electrical signals directly.

At present, the error testing technology has become more and more sophisticated. The bit error tester produces data and then receives it to calculate the error rate. There are many types to choose from. In this experiment, we adopt the CMR-2048V bit error rate tester, which is produced by Beijing Wangyuan Communication Co., Ltd. It has a laser transmitter with adjustable wavelength. In the previous work, we have used this equipment and platform to do experiments. For more details, refer $[9,10]$.

In the experiment, we control the frequency and amplitude of the vibrating mirror through the rotary axis control system. The experiment consists of 4 steps. First, the frequency is set to $50 \mathrm{~Hz}$ and the amplitude is set to $25 \mathrm{urad}$, $50 \mathrm{urad}, 75 \mathrm{urad}, \ldots, 300 \mathrm{urad}$. In each test, the time is set to 3 minutes and the laser wavelength is set to $850 \mathrm{~nm}$. The data rate is set to $10 \mathrm{Mbps}$. Later, the frequency is set to $100 \mathrm{~Hz}$ to do the experiment again. In the third step, the amplitude is set to 50 urad and the frequency is set to $10 \mathrm{~Hz}, 20 \mathrm{~Hz}$, $30 \mathrm{~Hz}, \ldots, 120 \mathrm{~Hz}$. In the fourth step, the amplitude is set to 100 urad to do the experiment again. Finally, the results are shown in Figures 7-10.

From Figure 7, we can see when the frequency is set to $50 \mathrm{~Hz}$, the amplitude is changeable, and $\lg (\mathrm{BER})$ of the ordinary receiver is up to nearly -8.5 . It means the BER is about $10^{-8.5}$. " $\lg (\mathrm{BER})$ " means " $\log _{10}(\mathrm{BER}) . "$ However, $\lg (\mathrm{BER})$ of the new receiver is nearly -9.2 . It means its BER is about $10^{-9.2}$. This result shows the BER of the new receiver is lower. In the experiment, when the amplitude is 0 , it means the vibrating mirror does not work. In this case, the $\lg (\mathrm{BER})$ of both new and ordinary receivers is about -11 .

From Figure 8, we can see $\lg (\mathrm{BER})$ of the ordinary receiver is up to nearly -8 . It means the BER is about $10^{-8}$. However, $\lg (\mathrm{BER})$ of the new receiver is nearly -9.1 . It 


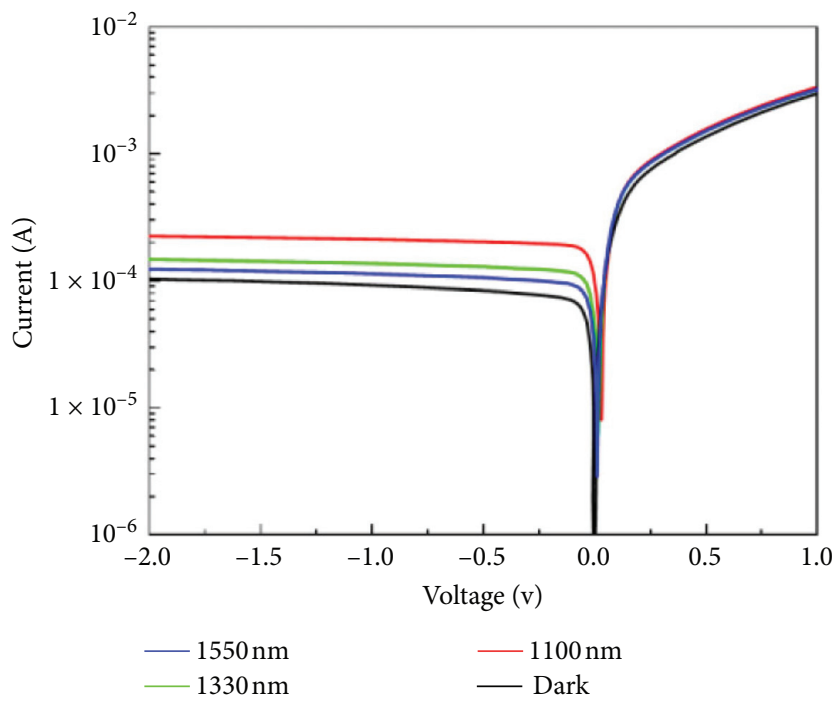

FIGURE 5: Dark and photo I-V curves under illumination of different wavelengths.

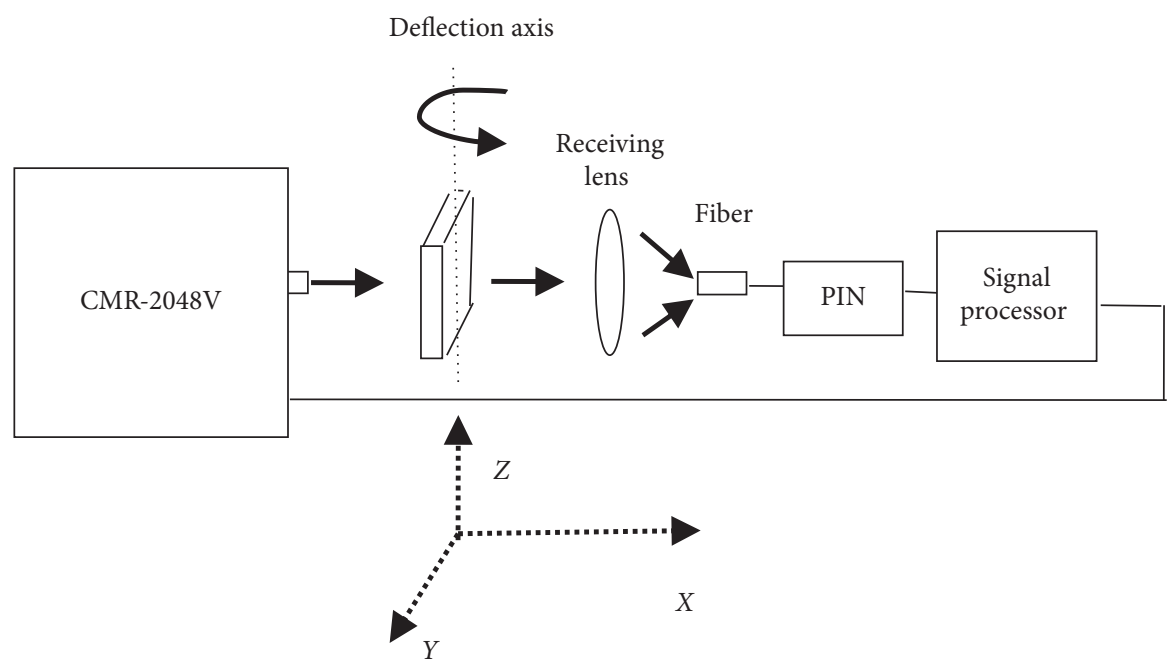

(a)

Deflection axis

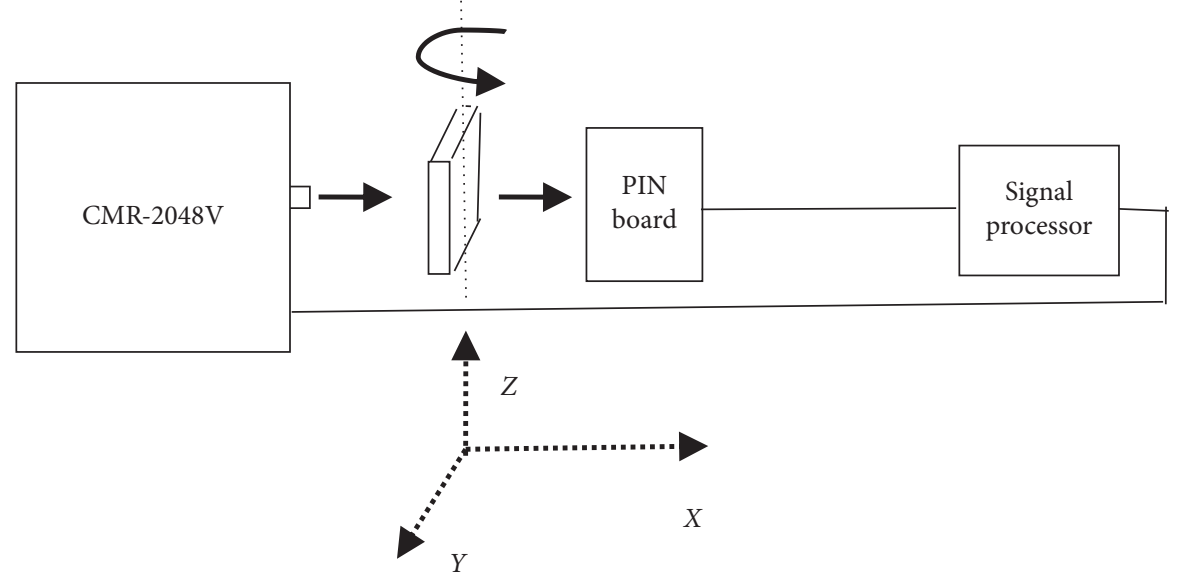

(b)

FIGURE 6: The experiment of bit error rate test: (a) adopting the typical receiving system; (b) adopting the new receiving system. 


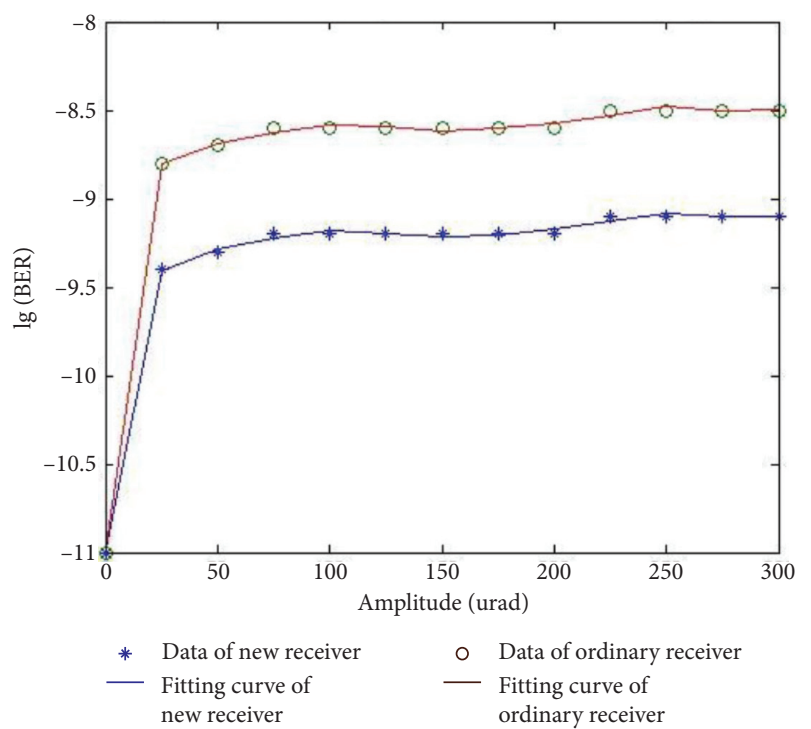

FIGURE 7: $\lg (\mathrm{BER})$ of both new and ordinary receivers (frequency: $50 \mathrm{~Hz}$ ).

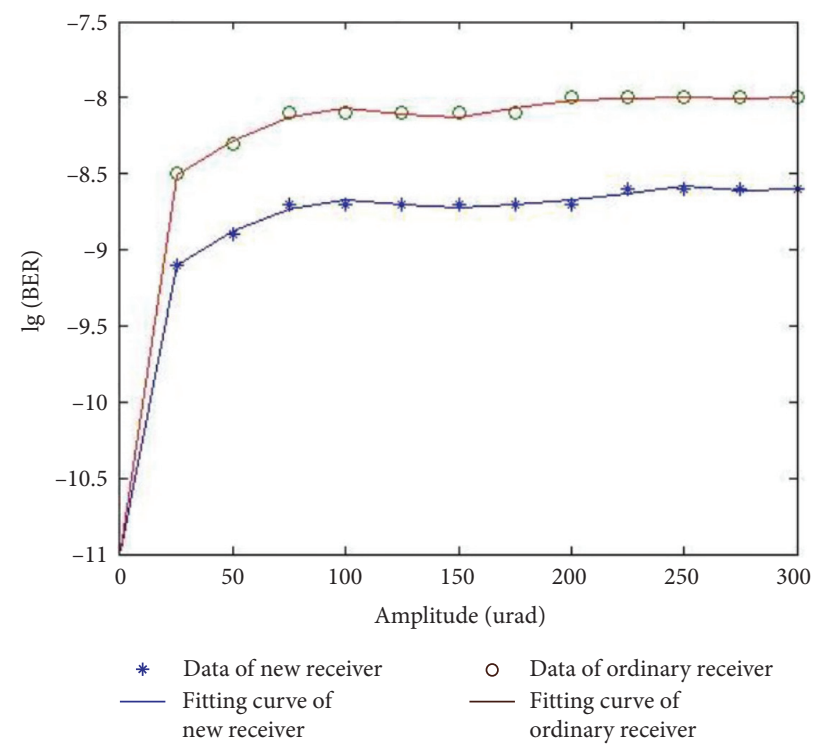

Figure 8: $\lg (\mathrm{BER})$ of both new and ordinary receivers (frequency: $100 \mathrm{~Hz}$ ).

means BER is about $10^{-9.1}$. Then, we can draw the similar conclusion that BER of the new receiver is lower. Compared with Figure 7, the difference is that when the frequency is set to $100 \mathrm{~Hz}, \lg (\mathrm{BER})$ of the ordinary receiver is up to nearly -8 , and it is higher than the value in the frequency of $50 \mathrm{~Hz}$. It means the BER is higher in a higher frequency. For the new receiver, we can draw the similar conclusion.

In Figure 9, the amplitude is set to $50 \mathrm{urad}$, and the frequency is changeable from 0 to $120 \mathrm{~Hz}$. $\lg (\mathrm{BER})$ of the ordinary receiver is up to nearly -8 . It means the BER is about $10^{-8}$. However, $\lg (\mathrm{BER})$ of the new receiver is nearly -9.1. It means the BER is about $10^{-9.1}$. In Figure 10 , the amplitude is set to $100 \mathrm{urad}$ and the frequency is changeable from 0 to $120 \mathrm{~Hz}$. $\lg (\mathrm{BER})$ of the ordinary receiver is up to nearly -7.9 . It means the BER is about $10^{-7.9}$. However, $\lg (\mathrm{BER})$ of the new receiver is nearly -9 . It means the BER is about $10^{-9}$. Both Figures 9 and 10 show the BER of the new receiver is lower.

All of the above figures show that when the vibrating mirror works, the BER of the new receiver is lower. When the vibrating mirror does not work, the BERs of both the ordinary and new receiver are almost the same. In the ordinary receiving system, the optical signals should be coupled into fiber by the receiving lens. However, the diameter of optical fiber is too small. It causes errors. In the vibration 


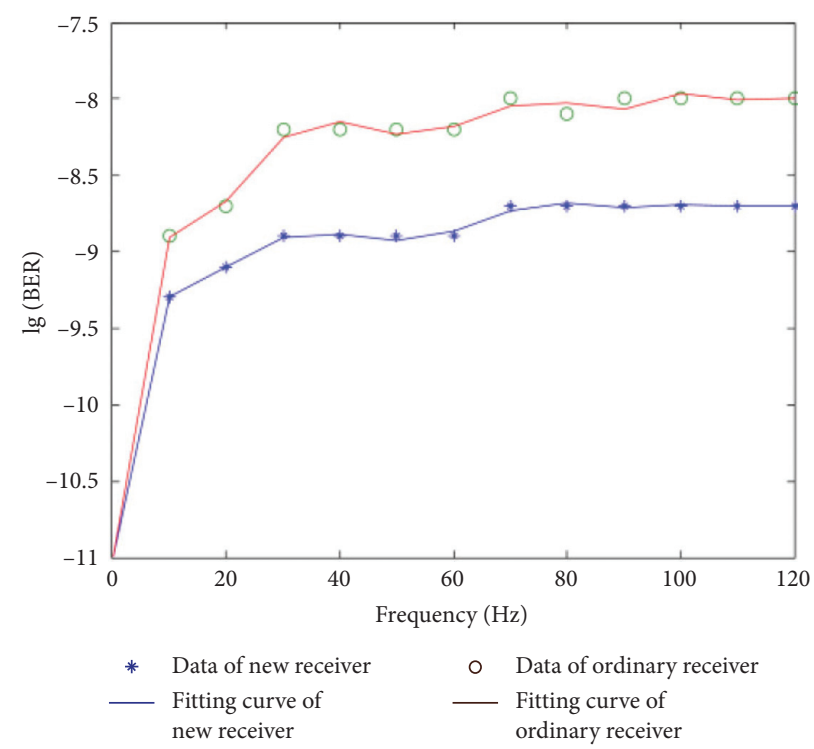

FIGURE 9: $\lg (\mathrm{BER})$ of both new and ordinary receivers (amplitude: $50 \mathrm{urad}$ ).

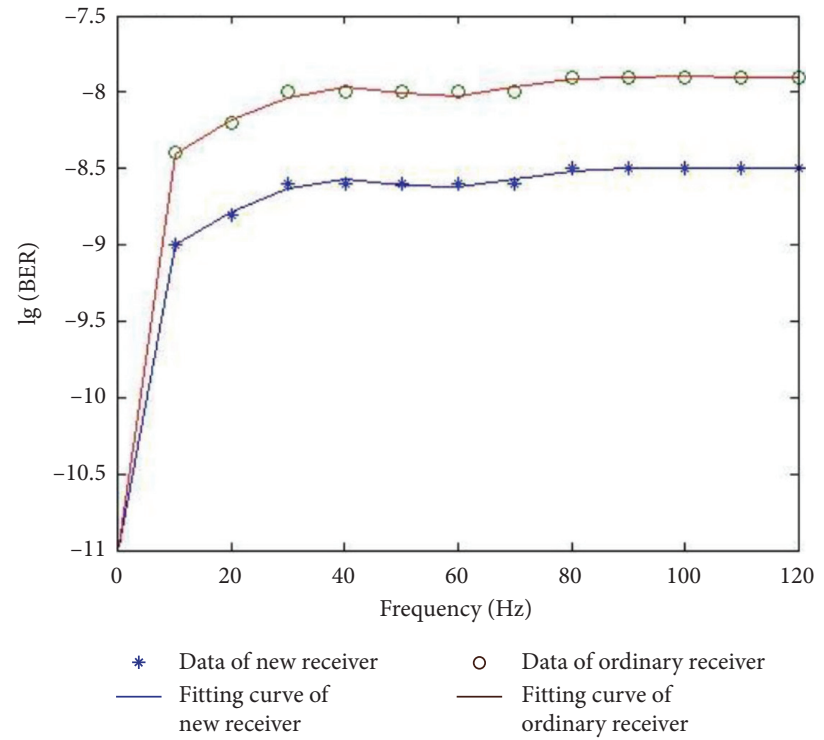

Figure 10: $\lg (\mathrm{BER})$ of both new and ordinary receivers (amplitude: 100 urad).

environment, this disadvantage is even obvious. In the new receiver, this shortcoming has been avoided.

Here, we would like to make the comparison with other studies. In [30], the performance evaluation of an FSO link has been performed using an array of photodetectors. From the results presented in [30], it can be concluded that as the number of photodetectors increased, there is a significant decrease in BER. Specifically, when single APD, array of 2 APD, array of 4 APD, and array of 8 APD are used at the receiver end of FSO link, the BERs are about $\left(10^{-8.2}, 10^{-8.5}\right.$, $10^{-9.2}$, and $\left.10^{-9.5}\right)$. For the new receiver in our work, we have carried out 4 groups of experiments. The experimental BERs are $\left(10^{-9.2}, 10^{-9.1}, 10^{-9.1}\right.$, and $\left.10^{-9}\right)$, respectively. It shows that the performance of our new receiver is equivalent to the array of 4 APD in [30].
In [31], the numerical simulation analysis showed that the NRZ coding and Mach-Zehnder modulation give the optimized overall performance. In this case, the BER is about $10^{-9.1}$. This value is equivalent to that of our new receiver. It means the performance of our new receiver reaches the optimized overall performance in [31].

In addition, in the experiment, the size of the PIN board is about $10 \times 8 \mathrm{~cm}^{2}$. In fact, we can connect several boards in series and in parallel to increase the receiving area. In this way, the difficulty in alignment could be further reduced.

The experiment is done in the dark room to avoid the effect of external light. However, in actual communication, the external light is ubiquitous. Then, how to avoid its influence needs further research. For this problem, we would like to propose two methods: one is the compensation 
method, and the other is fixed wavelength PIN receiver. For the first method, we detect the external light firstly and then deduct it out. For the second method, PIN receiver can only receive a certain kind of light, and it responds little to other light. Of course, it needs further study to judge whether such methods are effective. In the next step, we plan to study the relation between the size of the PIN board and the receiving efficiency.

\section{Conclusion}

In this work, we propose to use the PIN board as the new FSO receiver. Firstly, we use RTCVD and solar cell technology to produce the PIN board. Then, the PIN board is arranged as a new receiver in the FSO system to do a BER experiment. In the experiment, the vibrating mirror is set between the transmitter and receiver. We control the frequency and amplitude through the control system to simulate the different vibration environments. In total, we have carried out 4 groups of experiments. All the experimental results show that the new receiver performs better.

\section{Data Availability}

The data used to support the findings of this study are available from the corresponding author upon request.

\section{Conflicts of Interest}

The authors declare that there are no conflicts of interest.

\section{Acknowledgments}

The author HU Qinggui acknowledges the financial support from the National Natural Science Foundation of China (Grant no. 61275080), Solar Energy Integration Technology Popularization, and Application Key Laboratory of Sichuan Province (2018TYNSYS-Z-01). This project was supported by the National Natural Science Foundation of China (Grant no. 61275080) and the National Technology Innovation Fund (Grant no. 13C26212201166).

\section{References}

[1] S. Bhunia, M. Khan, and M. Yuksel, "In-band LOS discovery using highly directional transceivers," AD HOC Networks, vol. 91, no. 91, p. 101875, 2019.

[2] J. Sengupta, Z. Huang, J. Yao, and J. Z. Guo, “Adaptive digital combining for coherent free space optical communications with spatial diversity reception," Optics Communications, vol. 444, no. 444, pp. 32-38, 2019.

[3] Y. Ata, Y. Baykal, and M. C. Gökçe, "Effect of strong atmospheric non-Kolmogorov turbulence on the M-ary PSK subcarrier intensity modulated free space optical communications system performance," Applied Optics, vol. 58, no. 13, pp. 3639-3645, 2019.

[4] M. Smilic and Z. Nikolic, "Milic D etc., Comparison of adaptive algorithms for free space optical transmission in Malaga atmospheric turbulence channel with pointing errors," IET Communications, vol. 13, no. 11, pp. 1578-1585, 2019.

[5] Y. Ata, Y. Baykal, and M. C. Gökçe, "Effect of strong atmospheric non-Kolmogorov turbulence on the M-ary PSK subcarrier intensity modulated free space optical communications system performance," Applied Optics, vol. 58, no. 13, p. 3639, 2019.

[6] Q. Hu and C. Li, "The spatial light receiver and its coupling characteristics," Optics Communications, vol. 394, no. 394, pp. 18-22, 2017.

[7] H. Qinggui and M. Yining, "Tapered fiber array for free space optical communication," Journal of Communications Technology and Electronics, vol. 63, no. 10, pp. 1245-1249, 2018.

[8] H. U. Qinggui, "The new design of tapered fiber array for space optical receiver," Optical Materials, vol. 84, no. 84, pp. 367-370, 2018.

[9] $\mathrm{Q}$. $\mathrm{Hu}$ and $\mathrm{Y} . \mathrm{Mu}$, "Influence of vibration on the coupling efficiency in spatial receiver and its compensation method," Optical Engineering, vol. 57, no. 4, Article ID 046105, 2018.

[10] Q. Hu and C. Li, "The new tapered fiber connector and the test of its error rate and coupling characteristics," International Journal of Optics, vol. 2017, Article ID 2742709, 7 pages, 2017.

[11] H. U. Qinggui and M. U. Yining, "PIN photodiode array for free-space optical communication," Photonic Network Communications, vol. 36, no. 2, pp. 224-229, 2018.

[12] H. Kaushal and G. Kaddoum, "Optical communication in space: challenges and mitigation techniques," IEEE Communications Surveys \& Tutorials, vol. 19, no. 1, pp. 57-96, 2017.

[13] G. Xu and Z. Song, "Effects of solar scintillation on deep space communications: challenges and prediction techniques," IEEE Wireless Communications, vol. 26, no. 2, pp. 10-16, 2019.

[14] H. E. Nistazakis, A. N. Stassinakis, and H. G. Sandalidis, "QAM and PSK OFDM RoFSO over," IEEE Photonics Journal, vol. 7, no. 1, p. 7900411, 2015.

[15] K. W. Tombras, Q. Hao, and H. P. Zeng, "All-optical highprecision repetition rate locking of an $\mathrm{Yb}$-doped fiber laser," IEEE Photonics Technology Letters, vol. 27, no. 8, pp. 852-855, 2015.

[16] Y. Lin, Y. Ai, S. Xin et al., "Simulation of two-dimensional target motion based on a liquid crystal beam steering method," Optics Engineering, vol. 54, no. 5, Article ID 056102, 2015.

[17] S. G. Lee, C. J. Choi, and K. H. Shim, "The low temperature epitaxy of strained GeSn layers using RTCVD system," Electronic Materials Letters, vol. 14, no. 2, pp. 207-213, 2018.

[18] C. Park, D. Yoo, S. Im et al., "Large-scalable RTCVD Graphene/PEDOT: PSS hybrid conductive film for application in transparent and flexible thermoelectric nanogenerators," $R S C$ advances, vol. 7, no. 41, pp. 25237-25243, 2017.

[19] K. Sunilkumar, N. Anand, S. K. Satheesh et al., "Performance of free-space optical communication systems: effect of aerosol-induced lower atmospheric warming," Optics Express, vol. 27, no. 8, pp. 11303-11311, 2019.

[20] K. Prabu and D. S. Kumar, "MIMO free-space optical communication employing coherent BPOLSK modulation in atmospheric optical turbulence channel with pointing errors," Optical Communication, vol. 343, pp. 188-194, 2015.

[21] V. Torres-Company, J. Schroder, A. L. Fulop et al., "Laser frequency combs for coherent optical communications," Journal of Lightwave Technology, vol. 37, no. 7, pp. 1663-1670, 2019. 
[22] J. Kwangyun and K. Jungwon, "All-fibre photonic signal generator for attosecond timing and ultralow-noise microwave," Scientific Report, vol. 5, p. 16250, 2015.

[23] Ai Yun, A. Mathur, M. Cheffena et al., "Physical layer security of hybrid satellite-FSO cooperative systems," IEEE Photonics Journal, vol. 11, no. 1, pp. 1-14, 2019.

[24] A. E. Rakhshani, Journal of Applied Physics, vol. 108, pp. 094502-1-094502-4, 2010.

[25] L. C. Gaetano, "Dom assanto, fulgoni, lee nash," Journal of Lightwave Technology, vol. 26, pp. 2954-2959, 2008.

[26] E. F. Schubert, Light-emitting Diodesp. P88, second edition, Cambridge University Press, Cambridge, England, 2006.

[27] T. N. Ng, R. A. Lujan, S. Sambandan et al., Applied Physics Letters, vol. 91, pp. 063505-1-063505-3, 2007.

[28] T. N. Ng, W. S. Wong, L. Michael et al., Applied Physics Letters, vol. 92, pp. 213303-213311, 2008.

[29] G. I. Ayzenshtat, M. A. Lelekov, and O. P. Tolbanov, Semiconductors, vol. 42, pp. 443-447, 2008.

[30] M. Singh, "Evaluation of FSO link using array of photodetectors," Journal of Optical Communications, vol. 38, no. 43, pp. 249-254, 2017.

[31] M. A. Ahmed and K. K. Hasan, "Optimizing power level and bit error rate in free space optical communication," Telecommunications and Radio Engineering, vol. 79, no. 8, pp. $667-675,2020$. 